\title{
Search for vector-like quarks and excited quarks at CMS
}

\author{
Giorgia Rauco* on behalf of the CMS Collaboration \\ Universität Zürich \\ Winterthurerstrasse 190, 8057 Zürich \\ E-mail: giorgia.raucodcern.ch
}

\begin{abstract}
We present the results of the latest searches for new hypothetical heavy quarks using protonproton collisions data collected with the CMS detector at the CERN LHC at a center-of-mass energy of $13 \mathrm{TeV}$. Vector-like quarks are postulated to solve the hierarchy problem and stabilize the Higgs mass and they are not constrained by the Higgs discovery and electroweak measurements, as for the case of a fourth generation of fermions. They can either be produced singly or pair-wise and their decays result in a variety of final states, containing massive standard model quarks and bosons $(\mathrm{Z}, \mathrm{W}, \mathrm{H})$. Being these new particles expected to be appearing at the $\mathrm{TeV}$ scale, they give rise to boosted topologies, in which jet substructures techniques play a fundamental role. An alternative type of heavy quark resonance are the excited quarks, which are predicted by the compositeness model, being their evidence a clear signature of the composite structure of the ordinary matter. Their decay leads to the corresponding ordinary quark and a gluon, but also radiative transitions and decay in electroweak bosons are possible. The search for these new particles relies on the identification of an eventual excess of data on top of steeply falling background; therefore an optimal control of the backgrounds is fundamental.

Exclusions limits are set on the production cross sections for vector-like and excited quarks, considering several final signatures and multiple theoretical benchmarks.
\end{abstract}

The European Physical Society Conference on High Energy Physics

5-12 July, 2017

Venice

${ }^{*}$ Speaker. 


\section{Introduction}

The introduction of new hypothetical heavy quarks, whose left- and right-handed chiralities transform in the same way under the standard model (SM) gauge group $S U(3)_{c} \times S U(2)_{L} \times U(1)_{Y}$, might address some of the problems related to the nature of electroweak symmetry breaking, such as the hierarchy problem. These particles are called vector-like quarks (VLQs) [1] and can be produced pair-wise or singly. The possible decay modes involve electroweak and Higgs bosons, in addition to massive SM quarks.

Alternative new physics models, such as the compositeness model, predict the existence of another type of heavy quark resonances: the excited quarks [2]. At the LHC excited quarks would be produced predominantly in their single production through a gluonic excitation of the quark, but also other processes such as quark-antiquarks annihilation and gluon-gluon fusion are possible. They would then decay into the corresponding SM quark and a gauge boson (g, W, Z, $\gamma$ ).

We present here the latest searches performed using a data sample collected in 2016 by the CMS experiment [3] at the LHC in proton-proton collisions at a centre-of-mass energy of $\sqrt{s}=13$ $\mathrm{TeV}$, corresponding to an integrated luminosity of $35.9 \mathrm{fb}^{-1}$. In Section 2 we focus on the vectorlike quarks, while Section 3 is dedicated to excited quarks.

\section{Vector-like quarks}

\subsection{Search for VLQ B decaying with a b quark and a Higgs boson in the all jets final state [4]}

We present the search for VLQ B decaying in a bottom quark and a Higgs boson, with the Higgs decaying with a pair of $b$ quarks. The final state of this analysis is fully hadronic. Grooming techniques are employed to identify the Higgs jet candidate, allowing the $\mathrm{B}$ mass reconstruction $\left(\mathrm{m}_{\mathrm{B}}\right)$ as the invariant mass between the $\mathrm{H}$ boson and the $\mathrm{p}_{\mathrm{T}}$-leading $\mathrm{b}$ tagged jet not overlapping with the Higgs. Being the presence of a jet lying in the forward pseudorapidity region of the detector a peculiar feature of the single production process, to increase the analysis sensitivity events are split in two categories depending if at least one forward jet (with $|\eta|>2.4$ ) is reconstructed. The main background is the overwhelming multijets background, which is estimated from data in signal depleted control regions. Minor contributions, arising from $\mathrm{t} \overline{\mathrm{t}}, \mathrm{W}+\mathrm{jets}$ and $\mathrm{Z}+\mathrm{jets}$ processes, are taken from Monte Carlo simulation.

No excess of data over the SM predictions is observed and upper limits on the production cross section times branching ratio are computed. As theoretical benchmark, the singlet B hypothesis with $\mathscr{B}(\mathrm{B} \rightarrow \mathrm{bH})=25 \%$ and a coupling between the $\mathrm{b}$ quark and $\mathrm{Z}$ equal to 0.5 are assumed. A binned maximum likelihood fit is performed on the $\mathrm{m}_{\mathrm{B}}$ distribution and the systematical uncertainties are treated as nuisance parameters. The post-fit distribution of $m_{B}$ is shown in Figure 1. Expected and observed 95\% C.L. are also presented in Figure 1 for the range of resonance mass considered under the narrow width approximation. More realistic scenarios of wide-width resonances are also investigated and exclusion limits for the 10\%, 20\%, and 30\% width/mass possibilities are computed. 

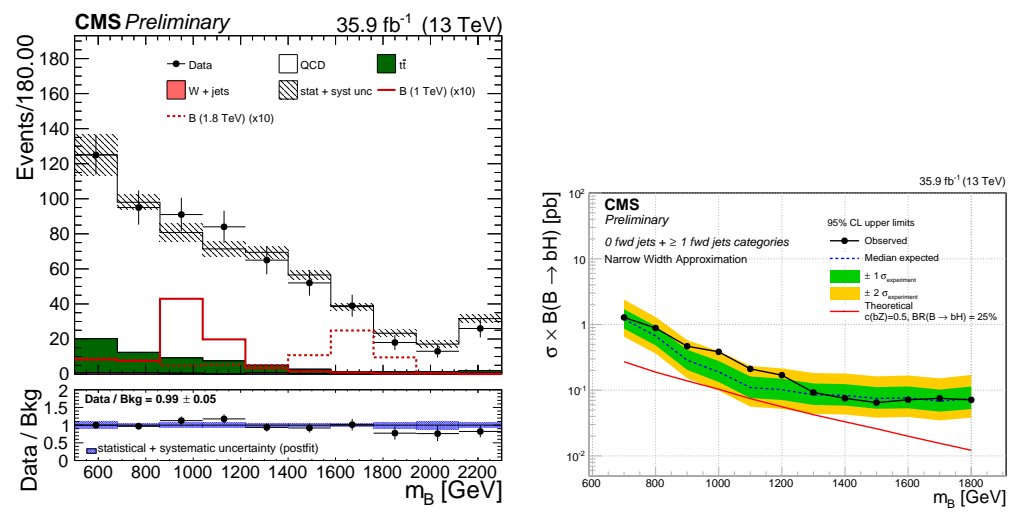

Figure 1: Post-fit distribution for the reconstructed VLQ B mass distribution in data and in the estimated background considering the category with at least one forward jet and with the harder selection on the hadronic activity applied (left) [4]. Observed and expected 95\% CL upper limit on the product of cross section and branching fraction for the singlet B, considering the B with a negligible width (right) [4].

\subsection{Search for VLQ T decaying with a top quark and a leptonic $\mathrm{Z}$ boson [5]}

Also the vector-like quark $\mathrm{T}$ is searched in its single production mechanism and we focus here on the VLQ T decay with a top quark and a leptonically decaying Z boson. Events are split in several categories depending on the forward jets multiplicity and on the number of $\mathrm{W}$ tagged and top tagged reconstructed jets. The search relies on the T mass reconstruction, based on the identification through grooming techniques of the $\mathrm{Z}$ and top candidates. The major background is $\mathrm{Z}+\mathrm{jets}$, but also minor contributions arising from other backgrounds $(\mathrm{t} \overline{\mathrm{t}}+\mathrm{V}, \mathrm{tZq}$, $\mathrm{t} \overline{\mathrm{t}}$, single $\mathrm{t}$, and SM diboson production) are taken into account. To reduce the dependence on the simulation, a data-driven background estimate based on control samples is employed.

No excess of data over the background prediction is observed and upper limits on the production cross section times the branching ratio are set, both under the assumption of narrow width approximation but also in the case of non-negligible resonance width. Both the hypotheses of VLQ $\mathrm{T}$ singlet and doublet are investigated and former results are presented in Figure 2.

\subsection{Search for VLQ $T$ decaying with a b quark and a $\mathrm{W}$ boson [6]}

We present here the search for the vector-like quark $\mathrm{T}$ when it is produced in pair, focusing on the final state driven by one $\mathrm{W}$ boson decaying leptonically and the other decaying hadronically. The analysis relies on a first selection based on the lepton and jet kinematics, including also a dedicated W-tagging where the jet is deconstructed into its subjets which are then replaced by the matched jets. To suppress QCD multijets background, selections on missing transverse momentum and on the sum of jets and leptons momenta are also applied.

Events satisfying preliminary requirements are then submitted to the kinematic fit, performed by means of a $\chi^{2}$ minimization, which takes as inputs the missing transverse momentum and the measured momenta of the charged lepton and the jets. From the kinematic fit the reconstructed mass of the VLQ T, Mreco, is evaluated and its distribution is shown in Figure 3. A good agreement is found between the expected $\mathrm{M}_{\text {reco }}$ background distributions and the data, with no statistically 

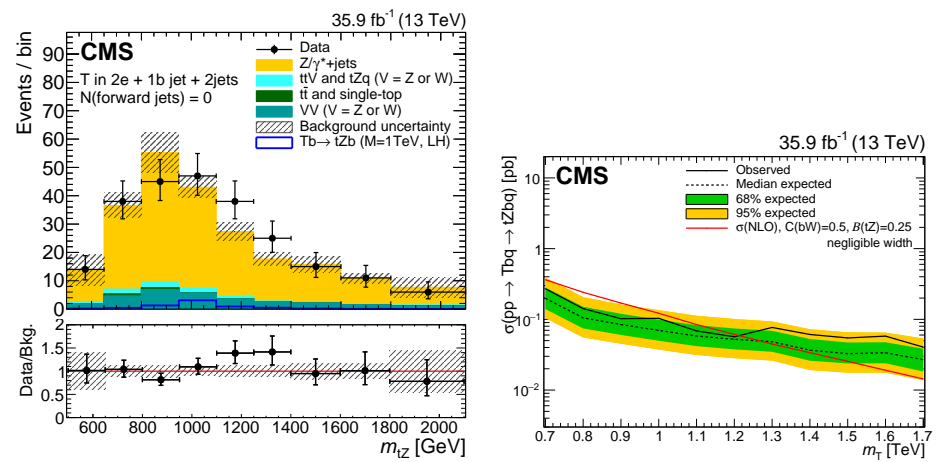

Figure 2: Reconstructed VLQ T mass distribution in data and in the background considering the electron channel, in the category where the top is reconstructed in its resolved topology and where zero forward jets are requested (left) [5]. Observed and expected 95\% CL upper limit on the product of cross section and branching fraction for the singlet LH T(b), where the T has a negligible width (right).

significant excess of events over background. Therefore, the results are used to set limits on the TT cross section and on the T mass, as shown in Figure 3.
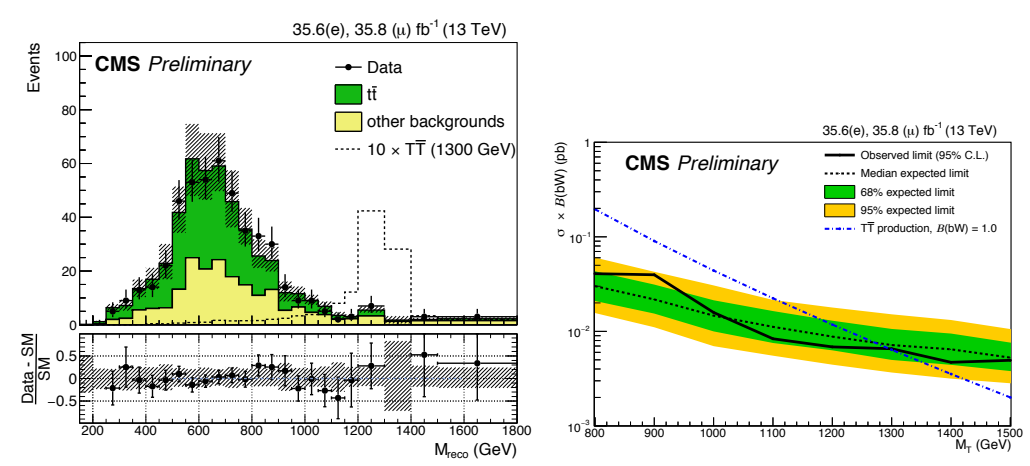

Figure 3: Post-fit distribution of $\mathrm{M}_{\mathrm{reco}}$ (left) and 95\% CL observed/expected exclusion limits (right) [6].

\subsection{Search for VLQ $\mathrm{X}_{-5 / 3}$ decaying in a $\mathrm{b}$ quark and a $\mathrm{W}$ boson $[7,8]$}

Vector-like quark $\mathrm{X}_{-5 / 3}$ can only decay with a $\mathrm{W}$ boson and a b quark and, depending on the decay mode of the $\mathrm{W}$ boson, several final states are possible. We consider here the single lepton signature and the one with at least two leptons with same sign, plus additional jets.

In the former we split the events in 16 categories, depending on the lepton flavor and on the W/top tagged jets multiplicity. The key variable on which this search is relying is the minimum invariant mass between the possible lepton-b jet pairs, which is the visible top quark mass as from reconstructed in the detector. Several backgrounds are considered, arising from QCD, electroweak and top processes. All the contributions are taken from simulation and are validated in signal depleted regions.

On the other hand, the search targeting final states with two same sign leptons exploits as key variable the hadronic leptonic activity, defined as the scalar sum of the jets and leptons transverse 
momenta. The background is mainly composed by SM processes with same sign prompt leptons but there are also minor contributions due to opposite sign prompt leptons and same sign events from fake lepton reconstruction. The former source is estimate from simulation, while the smaller backgrounds are described by means of data-driven techniques. No deviation from the SM expectations is observed in data and therefore upper limits at the 95\% C.L. are set on the production cross section, in both searches, as shown in Figure 4.
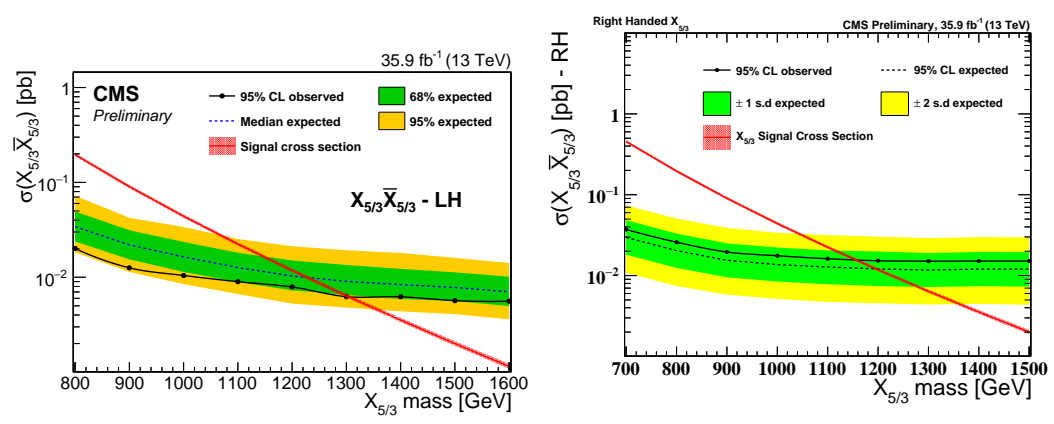

Figure 4: Expected and observed limits at the $95 \% \mathrm{CL}$ for a left-handed $\mathrm{X}_{5 / 3}$ in the single-lepton (left) and in the same-sign leptons (right) final state [7, 8].

\section{Excited quarks}

\subsection{Search for the production of $q^{*}$ and $b^{*}$ particles in events with photons and jets [9]}

A dedicated analysis in CMS looking for excited states of light quarks and b quarks is performed at CMS in the final state composed by one photon and a jet. This search is optimized to identify a data excess on top of the smoothly falling background. The event selection relies on mass and angular requirements, such as spatial distance and pseudorapidity opening between the photon candidate and the jet. The invariant mass of the $\gamma$ and jet system is modeled using an empirical parametrization with four parameters describing the background shape and normalization. Several tests are performed using alternative parametrization models to account for possible systematic bias due to the choice of parametric function, which resulted to be small as compared to the statistical uncertainty of the fit. In the mass region studied, no significant excess in data has been observed on top of the SM prediction and therefore the $\gamma+$ jet invariant mass spectra is used to set limits on the production cross-section of the $\mathrm{q}^{*} \rightarrow \mathrm{q} \gamma$ and $\mathrm{b}^{*} \rightarrow \mathrm{b} \gamma$, as shown in Figure 5. 

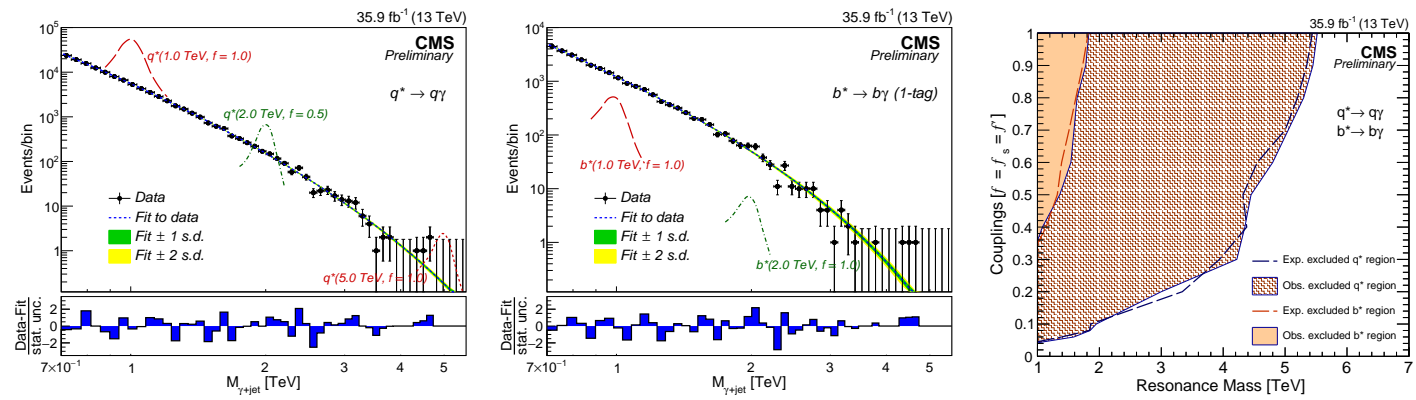

Figure 5: Invariant mass distribution of the $\gamma+$ jet system in data and background for the inclusive cate- gory in the $\mathrm{q}^{*}$ analysis (left) and for the $\mathrm{b}^{*}$ analysis in the category with one $\mathrm{b}$ tagged jet (center). Observed and expected excluded regions for $\mathrm{q}^{*}$ and $\mathrm{b}^{*}$ at $95 \% \mathrm{CL}$ as a function of the mass and the coupling (right) [9].

\section{References}

[1] J. A. Aguilar-Saavedra, "Identifying top partners at LHC", JHEP 0911, 030 (2009) doi:10.1088/1126-6708/2009/11/030 [arXiv:0907.3155 [hep-ph]].

[2] S. Bhattacharya, S. S. Chauhan, B. C. Choudhary and D. Choudhury, "Quark Excitations Through the Prism of Direct Photon Plus Jet at the LHC", Phys. Rev. D 80, 015014 (2009) doi:10.1103/PhysRevD.80.015014 [arXiv:0901.3927 [hep-ph]].

[3] CMS Collaboration, "The CMS experiment at the CERN LHC", JHEP 3, S08004 (2008)

[4] CMS Collaboration, "Search for a singly produced vector-like quark B decaying to a b quark and a Higgs boson in a fully hadronic final state using boosted topologies", CMS-PAS-B2G-17-009 CMS Physics Analysis Summary.

[5] A. M. Sirunyan et al. [CMS Collaboration], "Search for single production of a vector-like T quark decaying to a $\mathrm{Z}$ boson and a top quark in proton-proton collisions at sqrt(s) $=13 \mathrm{TeV}$ ", arXiv:1708.01062 [hep-ex].

[6] CMS Collaboration, "Search for vector-like quark pair production $\mathrm{T} \overline{\mathrm{T}}(\mathrm{Y} \overline{\mathrm{Y}}) \rightarrow \mathrm{bWbW}$ using kinematic reconstruction in lepton+jets final states at $\sqrt{s}=13$ TeV", CMS-PAS-B2G-17-003 CMS Physics Analysis Summary.

[7] CMS Collaboration, "Search for top quark partners with charge $5 / 3$ in the single-lepton final state at $\sqrt{s}=13$ TeV", CMS-PAS-B2G-17-008 CMS Physics Analysis Summary.

[8] CMS Collaboration, "Search for heavy vector-like quarks decaying to same-sign dileptons", CMS-PAS-B2G-16-019 CMS Physics Analysis Summary.

[9] CMS Collaboration, "Search for excited states of light and heavy flavor quarks in the $\gamma+$ jet final state in proton-proton collisions at $\sqrt{s}=13 \mathrm{TeV}$ ", CMS-PAS-EXO-17-002 CMS Physics Analysis Summary. 